\section{$\underset{\substack{\text { hommes } \\ \text { \& migrations }}}{ }$}

\section{Hommes \& migrations}

Revue française de référence sur les dynamiques

migratoires

1307 | 2014

L'Afrique qualifiée dans la mondialisation

\title{
L'insertion professionnelle des jeunes docteurs au Sénégal
}

\section{Bakary Doucouré}

\section{(apenEdition Journals}

\section{Édition électronique}

URL : http://journals.openedition.org/hommesmigrations/2884

DOI : 10.4000/hommesmigrations.2884

ISSN : 2262-3353

\section{Éditeur}

Musée national de l'histoire de l'immigration

\section{Édition imprimée}

Date de publication : 1 juillet 2014

Pagination : 87-92

ISBN : 978-2-919040-28-5

ISSN : $1142-852 X$

\section{Référence électronique}

Bakary Doucouré, «L'insertion professionnelle des jeunes docteurs au Sénégal », Hommes \& migrations [En ligne], 1307 | 2014, mis en ligne le 01 juillet 2017, consulté le 01 mai 2019. URL : http:// journals.openedition.org/hommesmigrations/2884; DOI : 10.4000/hommesmigrations.2884 


\title{
L'INSERTION PROFESSIONNELLE DES JEUNES DOCTEURS AU SÉNÉGAL
}

par BAKARY DOUCOURÉ, socio-anthropologue, chercheur au Conseil pour le développement de la recherche en sciences sociales en Afrique (Codesria).

\author{
Pour les jeunes diplômés sénégalais, l'insertion \\ professionnelle dans le monde académique et scientifique \\ relève d'un véritable parcours du combattant. Défaut \\ d'encadrement, manque de moyens financiers, mauvaise \\ gestion des institutions sont autant d'obstacles structurels \\ dont pâtit la recherche au Sénégal. Au-delà des seuls \\ diplômes, l'accès à l'élite universitaire passe par un long travail \\ de diffusion de la recherche sur la scène internationale \\ et la reconnaissance par les pairs.
}

L’enseignement supérieur et l'environnement de la recherche au Sénégal ont connu une dégradation, surtout depuis la fin des années 1980, comme le montrent de nombreux rapports ${ }^{1}$. Dans l'ensemble des cinq universités publiques du Sénégal, le personnel d'enseignement et de recherche est déficitaire en dépit des efforts budgétaires de créations de postes notées d'une année à l'autre. Pourtant, ce ne sont ni les besoins (exprimés par les universités), ni les demandes de recrutement (par les jeunes docteurs et chercheurs notamment) qui manquent. Par ailleurs, en dépit de la multiplication des instituts supérieurs de formation, voire de leur démultiplication incontrôlée, les jeunes docteurs et chercheurs continuent à faire face aux difficultés à s'insérer durablement dans les différentes structures.

Parmi les obstacles à l'insertion des jeunes diplômés dans l'enseignement supérieur et la recherche, on peut noter les effets des plans successifs des programmes d'ajustements structurels au début des années 1980. Ils ont entraîné un rétrécissement considérable des investissements et des

1. Ibrahima Hathie, État des lieux de la gouvernance de la recherche universitaire en Afrique de l'Ouest et du Centre, rapport de synthèse, Ottawa, CRDI, 2009 ; Ibrahima Hathie, État des lieux de la gouvernance universitaire. Cas de l'université Gaston-Berger de Saint-Louis, Ottawa, CRDI, 2009 ; Mouhamadou Sy, Rapport sur l'étude de la gouvernance universitaire à l'université Cheikh-Anta-Diop de Dakar, Dakar, CRDI, 2009 ; Académie nationale des sciences et techniques du Sénégal (ANSTS), "Rapport sur la situation de l'enseignement supérieur et ses exigences de qualité. Propositions d'actions et pistes prospectives", 2010 ; Syndicat autonome des enseignants du Sénégal (Saes), "Assises sur la normalisation du fonctionnement de l'enseignement supérieur du 1'er septembre au 8 décembre 2010", rapport, 2010 ; Oumar Sock, "Politique d'enseignement supérieur et de recherche scientifique au Sénégal. Situation actuelle et perspectives”, rapport, 2004 
fonds destinés à l'enseignement supérieur, ainsi que la désorganisation de la recherche. En outre, dans un contexte national où les emplois disponibles, notamment dans l'économie formelle, sont loin de satisfaire la demande 2 , les jeunes diplômés sont les plus exposés aux difficultés à trouver un emploi. C'est le cas des jeunes docteurs, directement ciblés dans cet article, pour qui l'obtention d'un emploi formel et durable reste un passage obligatoire dans leur volonté d'accéder à lélite académique et scientifique. À travers cette quête d'insertion et de reconnaissance, se dessinent les stratégies de construction de leur identité d'enseignants-chercheurs. La question de l'accès à lélite s'inscrit généralement dans la problématique des rapports entre le diplôme ou le titre, d'une part, et le statut socioprofessionnel ou le poste, d'autre part. Il s'agit notamment des rapports entre le système de

Les mobilités à caractère scientifique jouent un rôle prépondérant et crucial dans l'insertion durable des jeunes docteurs, en leur offrant des opportunités de se construire

une renommée au niveau international et de développer des réseaux relationnels et professionnels utiles à leur carrière scientifique.

cile l'accès à lélite, en dépit de l'obtention du doctorat. La notion d'élite, qui fait référence à "ceux d’en haut” dans un groupe social donné, ne peut être circonscrite de manière péremptoire tant il existe de multiples critères fondant la diversité des élites.

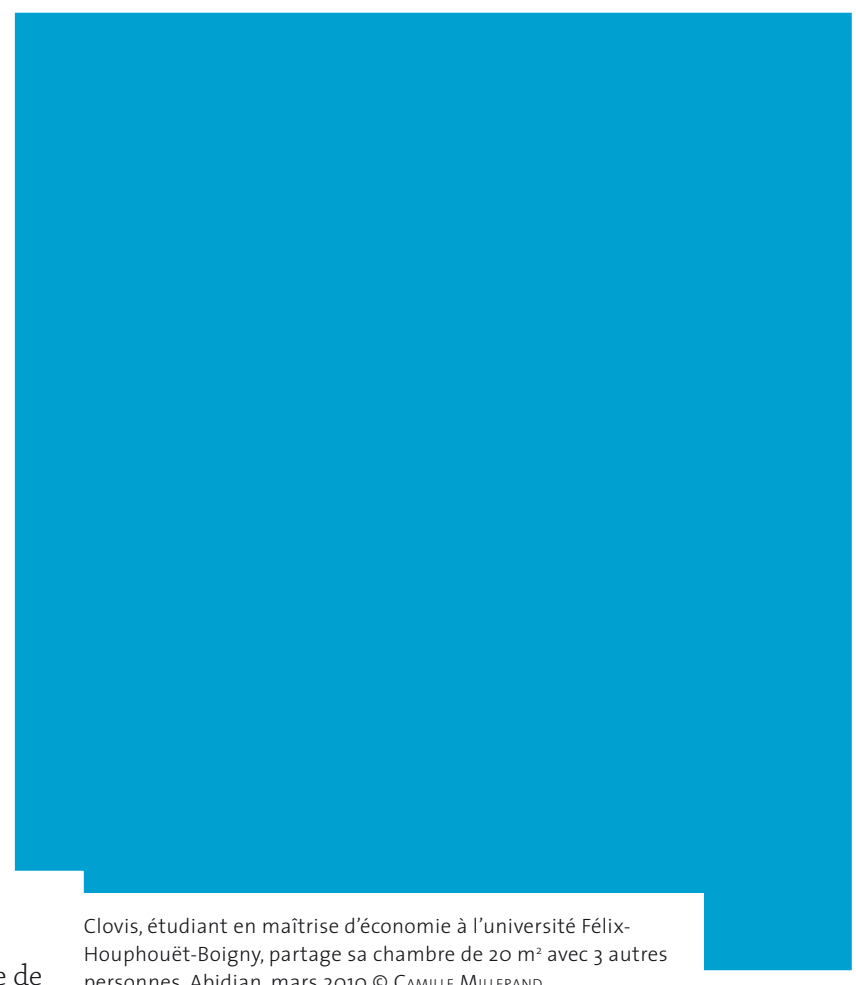

personnes. Abidjan, mars 2010 @ C CAmille Millerand

L'accès à l'élite reste toutefois une question importante pour les jeunes docteurs dans la mesure où linsertion ne se limite pas seulement à faire carrière : sa finalité est de faire une carrière scientifique exemplaire et reconnue par les pairs. En s'appuyant sur les données d'enquête du Projet jeunes chercheurs du Centre de recherche sur les politiques sociales (Crepos) et du Centre canadien de recherche pour le développement international $(\mathrm{CRDI})^{4}$, cet article analyse principalement les facteurs et les obstacles à linsertion des jeunes docteurs dans le monde académique et scientifique. Il en ressort principalement qu'à l'instar des productions scientifiques, les mobilités à caractère scientifique jouent un rôle prépondérant et cru- 
cial dans l'insertion durable des jeunes docteurs, en leur offrant des opportunités de se construire une renommée au niveau international et de développer des réseaux relationnels et professionnels utiles à leur carrière scientifique.

\section{"Jeunes docteurs", "élite académique" : définitions et marqueurs au Sénégal}

Les notions de "jeune docteur" et d"élite" ne sont pas consensuelles. Dans le cadre de cette étude, lâge de soutenance de la thèse et la durée écoulée entre la soutenance et l'enquête sont les deux critères retenus pour désigner les jeunes chercheurs. L'enquête s'est adressée aux diplômés, toutes disciplines scientifiques confondues, titulaires d'un doctorat de $3^{e}$ cycle ou d'un doctorat unique. L'âge plafond de 45 ans a été choisi, compte tenu des retards de soutenance, en partie liés au taux d'encadrement élevé (soit le nombre de doctorants par enseignant) et à l'absence de moyens financiers et logistiques nécessaires à la réalisation des recherches doctorales. À ce critère d'âge limitatif s'est ajouté celui du nombre d'années écoulées entre la soutenance et l'enquête. Ainsi, tous les docteurs de moins de 45 ans ayant soutenu leur thèse depuis moins de six ans font partie de la population cible. Le choix de cette durée s'explique par le fait que, dans le système du Conseil africain et malgache pour l'enseignement supérieur $\left(\mathrm{Cames}^{5}\right)$ qui régit l'accès aux différents grades ${ }^{6}$ dans les universités publiques sénégalaises, il faut un délai minimum de cinq ans pour accéder au titre de maître de conférences ${ }^{7}$, notamment dans les disciplines des sciences sociales et humaines, et dans les facultés des sciences et techniques. Par ailleurs, pour les disciplines où l'agrégation existe, il faut en général un délai de cinq ans pour passer l'agrégation, bien que ces disciplines offrent la possibilité de concourir pour l'agrégation une fois que l'on est recruté comme assistant. Or c'est le grade de maitre de conférences dans le domaine de l'enseignement, et celui de directeur de recherche dans les institutions de recherche, qui consacrent la maturité scientifique de l'enseignant-chercheur, et donc son statut de chercheur senior. Quant à l'élite académique et scientifique, elle s'appréhende à travers un certain nombre de marqueurs. À l'instar d'Aminata Diaw ${ }^{8}$, nous pensons que le statut d'intellectuel ${ }^{9}$ et celui de membre de l'élite académique et scientifique qui en découle ne peuvent être conférés uniquement par un diplôme ou un critère linguistique. Bien que déterminants, les marqueurs symboliques de l'appartenance à l'élite académique et scientifique ne peuvent être arrêtés de manière péremptoire. Il semble que la conscience d'appartenir à une même communauté de valeurs, l'exercice d'une réflexion critique sur soi-même et sur sa propre société (voire sur d'autres sociétés) ainsi que l'ouverture à l'Autre et aux autres cultures soient considérés comme faisant partie de ces marqueurs symboliques. Toutefois, il est difficile d'identifier catégoriquement les membres ou les prétendants au statut d'intellectuel tant les valeurs, les priorités et les démarches de chacun peuvent différer voire s'opposer.

\section{L'insertion problématique des jeunes docteurs}

L'insertion professionnelle des jeunes docteurs prend plusieurs formes : durable, temporaire ou précaire. L'insertion durable s'entend ici comme l'obtention d'un emploi à durée indéterminée dans une institution d'enseignement, de formation et/ ou de recherche. Elle offre au jeune chercheur la 
possibilité de faire carrière et de gravir les échelons permettant d'accéder à l'élite académique et scientifique. Deux exemples d'initiatives et de stratégies peuvent être mentionnés.

Tout d'abord, la production scientifique à travers des publications et des recherches postdoctorales constitue l'une des principales préoccupations des jeunes docteurs ayant choisi notamment la voie de l'enseignement universitaire et de la recherche. Ce critère reste déterminant lors des recrutements dans les universités, en particulier pour les postes permanents. Toutefois, les conditions de production scientifique demeurent difficiles pour la plupart des jeunes chercheurs. Les moyens financiers, techniques et logistiques nécessaires au développement d'activités de recherche et de production scientifique font souvent défaut. Au final, de nombreux jeunes docteurs éprouvent des difficultés à mettre en œuvre des recherches postdoctorales et à se faire publier de manière régulière.

Ensuite, les mobilités professionnelles de courte ou de longue durée (participation aux rencontres scientifiques au niveau national et international, voyages d'études à l'étranger, etc.) sont perçues par les jeunes docteurs comme autant d'atouts pour une insertion durable. En effet, les activités scientifiques (colloques, séminaires, conférences) menées dans le cadre des mobilités offrent aux jeunes docteurs des opportunités de vulgarisation de leurs travaux et d'intégration des réseaux académiques et de recherche. Cependant, beaucoup de jeunes docteurs au Sénégal font face à des contraintes qui réduisent leurs chances de participer à des rencontres scientifiques, surtout celles organisées à l'étranger. Parmi les obstacles, les possibilités limitées de prise en charge matérielle et financière. Au niveau des universités et des institutions de recherche publiques, par exemple, seuls les enseignants et les chercheurs permanents bénéficient de la prise en charge de leurs voyages d'études. Il n'existe aucun dispositif destiné à soutenir les jeunes docteurs pour de telles mobilités professionnelles.

\section{Des obstacles structurels et conjoncturels}

Plusieurs de ces obstacles d'ordre structurel sont mentionnés par les différents acteurs enquêtés. Les responsables institutionnels, par exemple, déplorent tout d'abord l'absence d'une politique de recherche cohérente. Elle se traduit par l'éclatement des structures chargées de la recherche, leur petit nombre tout comme la faiblesse des ressources financières, techniques et humaines dédiées à la recherche. L'absence d'une politique d'insertion des jeunes chercheurs renvoie à celle d'un système d'information sur l'emploi scientifique. En outre, d'autres facteurs sont aussi mentionnés : les difficultés de fonctionnement et la mauvaise gouvernance des institutions de recherche existantes; le statut public de certaines d'entre elles qui les privent des fonds nécessaires au recrutement des jeunes chercheurs.

Des obstacles d'ordre conjoncturel viennent souvent accentuer ces difficultés. L'enseignement supérieur privé au Sénégal en donne une bonne illustration. En effet, la multiplication et le développement des institutions privées d'enseignement supérieur et de formation à partir des années 1990 offrent une possibilité d'insertion aux jeunes docteurs. En 2010, près de 70 établissements privés d'enseignement et de formation bénéficiaient d'un agrément de l'État ${ }^{10}$. Cependant, les contrats proposés aux jeunes chercheurs en général (dont les jeunes docteurs) demeurent essentiellement précaires et de courte durée. Leurs missions dans ces établissements sont exclusivement centrées sur l'enseignement. Il n'existe pas encore de véritables pôles de recherche au sein de ces institutions privées. La situation financière ${ }^{11}$ de la plupart d'entre elles, les coûts liés à un recrutement durable et massif de jeunes docteurs, les charges de fonctionnement des établissements, la situation fortement concurren- 
tielle entre eux, créent une conjoncture peu favorable à l'insertion durable des jeunes chercheurs. En un mot, les contraintes du marché, ajoutées à celles d'ordre financier, légal, voire infrastructurel génèrent des réticences. Dans un tel contexte, l'insertion des jeunes se caractérise par une longue période d'attente qui compromet leurs chances, quand ils sont enfin recrutés, d'accéder aux grades universitaires et académiques les plus élevés.

\section{L'accès à l'élite académique : un processus cumulatif et non linéaire}

L'accès à l'élite est déterminé par une conjonction de facteurs à la fois scientifiques, professionnels et économiques qui rend complexe la compréhension du lien entre le diplôme, le titre académique et la reconnaissance socioprofessionnelle, chacun contribuant à l'assimilation à l'élite académique et scientifique. Il est le résultat d'une progression professionnelle et scientifique conduisant à des statuts, des fonctions, des titres, des distinctions, des identités nouvelles et une reconnaissance au sein d'une communauté scientifique dont on partage et reconnaît les valeurs et les symboles. L'accès à l'élite n'est pas un événement synchronique, il se construit au fil d'un processus fait de trajectoires complexes. Ce processus non linéaire ne peut être appréhendé à partir de l'exemple unique des jeunes docteurs qui, par essence, entament leur carrière académique et scientifique. Ainsi, pour mieux mettre en exergue le caractère dynamique de l'accès à lélite, il convient de se pencher sur la trajectoire de l'une de ses figures : Souleymane Bachir Diagne ${ }^{12}$.

Né en 1955 à Saint-Louis, à la fois philosophe et mathématicien, il poursuit ses études en France, pour faire hypokhâgne et khâgne au prestigieux lycée Louis-le-Grand de Paris. Il est reçu à l'agrégation de philosophie avant de rejoindre l'université d'Harvard (USA) dans le cadre d'un programme d'échanges. Il soutient sa thèse de doctorat de $3^{\text {e }}$ cycle à la Sorbonne (France) en 1982, puis sa thèse de doctorat d'État en 1988. Professeur successivement à l'université Cheikh-Anta-Diop de Dakar (Ucad, Sénégal), à l'université Northwestern (USA) et à l'université de Columbia (USA), il a occupé de multiples fonctions au cours de sa carrière. On relève notamment les fonctions d'assesseur à la faculté de lettres et sciences humaines de l'université de Au niveau des universités et Dakar, de conseiller spécial desinstitutions de recherche pour l'éducation et la culture publiques, par exemple, (de 1993 à 1999) du prési- seuls les enseignants dent de la République du Sé- et les chercheurs permanents négal, de directeur, codirec- bénéficient de la prise teur ou membre du comité en charge de leurs voyages scientifique ou de publica- d'études. II n'existe aucun tion de nombreuses revues ${ }^{13}$ dispositif destiné à soutenir les et d'institutions internatio- jeunes docteurs pour de telles nales ${ }^{14}$. Outre son abondan- mobilités professionnelles. te bibliographie ${ }^{15}$, il a reçu plusieurs distinctions et prix sur le plan national et international comme le prix Édouard Glissant en 2011. Aussi, la carrière professionnelle de Souleymane Bachir Diagne est marquée par une forte mobilité liée à ses activités académiques et scientifiques. Diagne n’est pas présenté ici comme un idéal type au sens wébérien du terme. Mais son exemple permet, au-delà de la diversité des parcours rencontrés, de dégager quelques marqueurs communs offrant un cadre d'analyse à la problématique de l'accès à l'élite pour les jeunes docteurs au Sénégal. Nous pouvons retenir : l'accès aux grades universitaires et scientifiques les plus élevés; la reconnaissance nationale et internationale au travers de distinctions scientifiques et de titres honorifiques; diverses formes 
de mobilités qui se traduisent par la nomination à des postes à responsabilités au niveau universitaire, administratif ou politique, et la participation à des rencontres scientifiques nationales et internationales. À ce titre, l'analyse du parcours professionnel des jeunes docteurs enquêtés révèle qu'ils ne peuvent encore être associés à l'élite définie comme un groupe très restreint, auquel on attribue une valeur supérieure dans un milieu déterminé. Un double processus de reconnaissance par les pairs et de progression individuelle ouvre la voie aux fonctions et responsabilités les plus élevées sur le plan académique, administratif ou politique, tout en conférant à l'enseignant et/ou au chercheur un statut d'élite. L'accès à l'élite académique et scientifique n'est donc pas déterminé par la seule obtention du doctorat, il se construit au fil de la carrière. En définitive, les jeunes docteurs se situent davantage dans la communauté académique et scientifique qu’au niveau de l'élite.

\section{Conclusion}

La question de l'élite en tant que groupe reste complexe : il n'existe ni homogénéité des profils et des parcours, ni consensus sur le plan idéologique (ni même des valeurs). Il est d'ailleurs préférable de parler des élites académiques et scientifiques plutôt que d'une seule et unique élite. Celle-ci, considérée individuellement ou collectivement, résulte d'une construction à la fois théorique et empirique. L'approche théorique met plutôt en exergue les opinions, les idéologies, les valeurs, l'engagement intellectuel et scientifique, etc., qui distinguent les élites du reste de la communauté. L'approche empirique ou pragmatique, quant à elle, privilégie des critères et des facteurs plus concrets et de nature institutionnelle (diplômes, grades, titres, distinctions, postes, fonctions et responsabilités). Cependant, quel que soit le type d'approche privilégié, la notion d'élite consacre une distinction réservée à une minorité en vertu de qualités (exceptionnelles, spécifiques ou propres) ou tout simplement de marqueurs dont ne jouissent pas la majorité et le reste de la communauté. Pour cela, l'élite académique et scientifique, au sens collectif, désigne un groupe très restreint d'enseignantschercheurs situés en haut de l'échelle de la sphère académique et scientifique, dont la position est reconnue comme telle par au moins une partie de la communauté scientifique et se manifeste à travers un certain nombre de fonctions, de distinctions et de titres notamment.

L'accès à l'élite est le résultat d'un processus individuel de progression et d'évolution sur le plan académique et scientifique qui conduit à une reconnaissance professionnelle et sociale à travers des processus d'intégration, de nomination et de cooptation dans les instances académiques, scientifiques, administratives, voire politiques les plus élevées et les plus prestigieuses. En tant que processus, l'accès à l'élite requiert du temps et de ce fait ne peut pas s'opérer dès le début de la carrière. Pour les futures et potentielles élites, les diverses formes de mobilités (estudiantines et professionnelles) contribuent à la fois à leur formation (acquisition, renouvellement et renforcement des connaissances, obtention de diplômes) et à la constitution de réseaux utiles au développement de leur carrière. En plus des productions scientifiques et de la mobilité internationale, d'autres stratégies d'arrangement, de contournement ou d'intériorisation sont mobilisées et mises en œuvre par les jeunes docteurs. On peut mentionner parmi celles-ci le passage par le statut d'enseignant vacataire avant l'obtention d'un poste permanent ; la pratique de la consultance à titre individuel ; les initiatives d'autoemploi par la création de cabinets de consultance ou de bureaux d'étude et de recherche ; la création d'associations dans l'espace universitaire pour défendre leurs intérêts. Le développement de ces stratégies se fait aussi dans un cadre élargi aux autres jeunes chercheurs, tout en ayant recours à l'appui des collègues durablement insérés et même des acteurs extérieurs au milieu universitaire et académique. 